\title{
Characteristics of the Diurnally Varying Electron Flux near the Polar Cap
}

\author{
Martin H. ISRAeL ${ }^{1}$ AND Rochus E. Vogt \\ California Institute of Technology, Pasadena, California 91109
}

\begin{abstract}
We report on the characteristics of the diurnally varying electron flux observed near the polar cap. The discussion is based on balloon observations, made near Fort Churchill, Manitoba, in 1967, of both the electron flux vertically incident on the atmosphere and the electron splash albedo. We measured an electron splash albedo flux below $100 \mathrm{Mev}$ equal to the flux of electrons below $100 \mathrm{Mev}$ incident on the atmosphere during daytime. The observed diurnal variations of the flux of electrons with energies $\$ 100 \mathrm{Mev}$ is consistent with geomagnetic cutoff variations calculated for models of the magnetosphere. We deduce a geomagnetic ctuoff rigidity of $\lesssim 17 \mathrm{Mv}$ for the nighttime interval and between 70 and $110 \mathrm{Mv}$ for the daytime interval at an invariant latitude near $68^{\circ}$ in 1967.
\end{abstract}

\section{INTRODUCTION}

Many of the observations of cosmic-ray electrons in the past have been performed on balloon payloads and were subject to atmospheric and geomagnetic influences. Although the measurements of many investigators show reasonable agreement for electron fluxes at energies above several hundred Mev, the quoted results at lower energies are considerably more uncertain. Early measurements of electron fluxes below about $100 \mathrm{Mev}$ [Meyer and Vogt, 1961; L'Heureux, 1967; Beedle and Webber, 1968] contained a (then unknown) significant component of return albedo electrons. The study of the diurnal variation of low-energy electron fluxes by Jokipii et al. [1967] and the later work by Webber [1968] and Israel and Vogt [1968] produced more accurate primary-flux values by identifying the daytime component of the diurnally varying electron flux at the top of the atmosphere as return albedo and the nighttime component as primaries. In this paper we shall present further evidence, based on our 1967 observations of the diurnal variation of electron fluxes near the polar cap and direct measurements of the electron albedo, to support the interpretation of the diurnal variation first proposed by Jokipii et al. [1967]. We also present evidence for a latitude and energy dependence

\footnotetext{
1 Present address: Department of Physics, Washington University, St. Louis, Missouri 63130.
}

of the time of the night-day transition in the electron flux.

\section{Instrument and Balloon Flights}

The detector system used in these observations was a scintillation-counter telescope, which included a gas Cerenkov counter, a spark chamber with lead plates, and a scintillation counter for energy determination. This system is described in the accompanying paper [Israel, 1969], which hereafter will be referred to as paper 1 .

The data reported in this paper are derived from four balloon flights of this detector. The balloons were launched from Fort Churchill, Manitoba, in June and July 1967. Table 1 of paper 1 summarizes pertinent flight conditions. On flights $\mathrm{C} 1, \mathrm{C} 2$, and $\mathrm{C} 4$ the detector telescope pointed toward the zenith. During flight C3 the detector was inverted to look at the upward-moving splash albedo.

Contours of constant geomagnetic cutoff rigidity in the Churchill vicinity are shown in Figure 1. Also shown are the trajectories of the four flights. The cutoffs in this figure were calculated by means of a spherical harmonic expansion of the earth's internal magnetic field [Shea et al., 1968]. The true cutoffs are lower than these values and vary with local time as shown in this paper.

All flights occurred during quiet geomagnetic conditions, with $K p$ below 3 [Lincoln, 1968]. The daily averages of the Mount Washington 


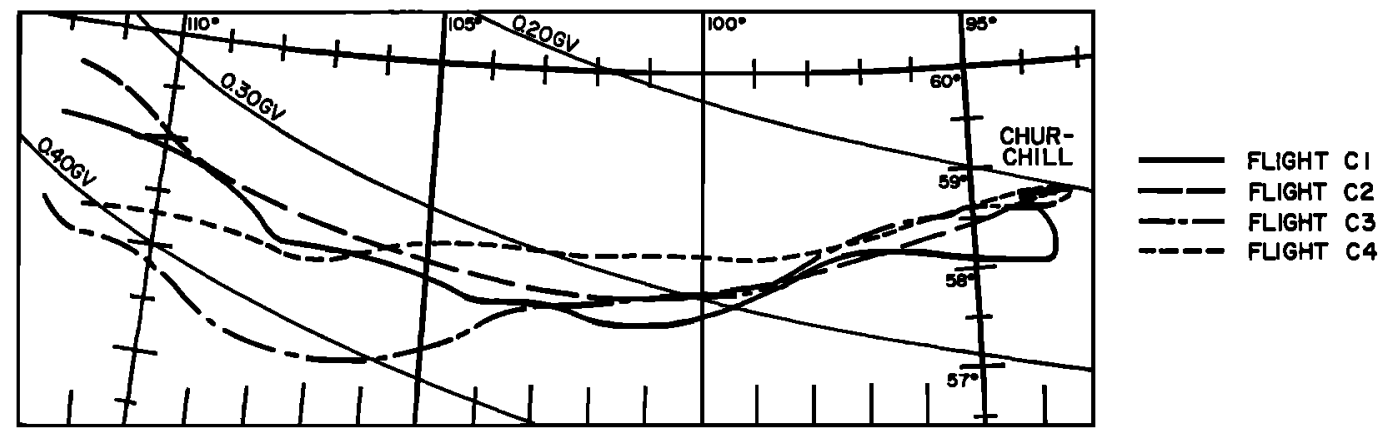

Fig. 1. Trajectories of the balloon flights and contours of constant geomagnetic cutoff rigidity. Cutoffs are derived from the internal geomagnetic field only.

neutron monitor count rate during all flights differed by less than $2 \%$ from that of flight $\mathrm{C} 1$ (J. A. Lockwood, private communication). Further evidence that these flights occurred during quiet times comes from the solar-proton monitor on the Explorer 34 satellite [ESSA, 1968]. No solar protons with energy above $30 \mathrm{Mev}$ were detected outside the magnetosphere from June 9, a week before flight C1, through the end of July. A barely detectable flux of 10Mev protons, less than 0.2 particle $/ \mathrm{cm}^{2}$ sec ster, was present during flight $\mathrm{C} 1$, but none was detected at the dates of our other flights.

\section{OBSERVATIONS}

Figure 2 shows the event rate of low-energy electrons observed at float altitude as a function of local time. The clearest evidence of a diurnal flux variation occurs in these events of type 1, which represent electrons of approximately 12 to $50 \mathrm{Mev}$ at the detector. (The discussion of energy determination and event types is given in paper 1.) The first three curves of Figure 2 represent the rate of type 1 events during the float periods of flights $\mathrm{C} 1, \mathrm{C} 2$, and $\mathrm{C} 4$, in which the detector was oriented toward the zenith. The dashed curves indicate the atmospheric secondary contribution as discussed in paper 1. Variations in the secondary flux reflect changes in the balloon altitude.

The first two intervals plotted for flight $\mathrm{C} 1$ represent data gathered during the last 70 minutes of ascent. They are included in this plot to indicate the morning transition, which fortuitously occurred just as the balloon reached float altitude. This time is the latest recorded occurrence of the morning step, and this flight is the first to record both a morning flux increase and an evening flux decrease. In the data of flights $\mathrm{C} 2$ and $\mathrm{C} 4$ the morning step is also apparent, but it occurs earlier and less sharply than in C1. Flights $\mathrm{C} 2$ and $\mathrm{C} 4$ were terminated before the evening transition.

For comparison, the bottom curve in Figure 2 gives the rate of type 1 events observed during flight $\mathrm{C} 3$, when the detector was oriented toward the nadir to observe splash albedo. This plot displays no transition comparable to the steps during the other flights. We expect no

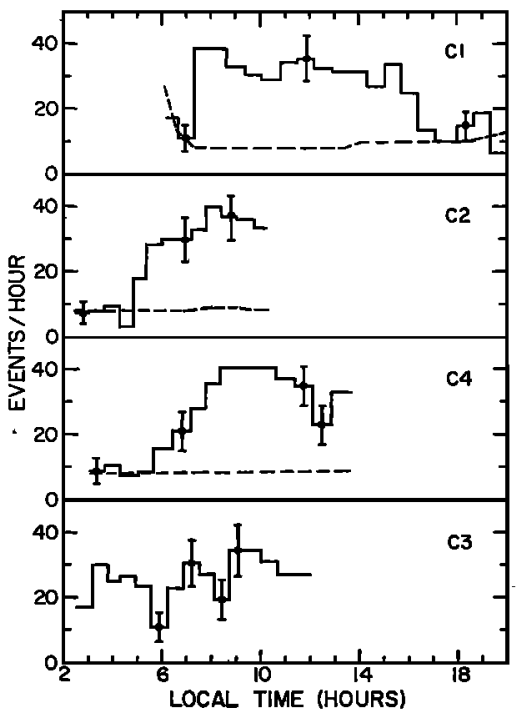

Fig. 2. Type 1 event rate versus local time at the detector, during flights $\mathrm{C1}, \mathrm{C} 2, \mathrm{C4}$, and C3. Solid line indicates observed event rate, and error bars indicate typical statistical errors. Dashed line represents calculated rate due to atmospheric secondary electrons. 
TABLE 1. Electron-Event Rates

Rates are expressed as events per hour. Only statistical uncertainty is indicated.

\begin{tabular}{|c|c|c|c|c|c|}
\hline \multirow{2}{*}{$\begin{array}{l}\text { Event } \\
\text { Type }\end{array}$} & \multirow{2}{*}{ Interval* } & \multicolumn{3}{|c|}{ Flight } & \multirow[b]{2}{*}{ C3 } \\
\hline & & C1 & C2 & $\mathrm{C} 4$ & \\
\hline \multirow[t]{2}{*}{$0 \dagger$} & $\mathbf{N}$ & & & $45.1 \pm 4.4$ & \\
\hline & D & & & $59.4 \pm 3.6$ & \\
\hline \multirow[t]{3}{*}{1} & $\mathbf{N}$ & $10.7 \pm 1.9$ & $8.1 \pm 2.0$ & $8.7 \pm 1.9$ & $\cdots$ \\
\hline & D & $33.0 \pm 1.9$ & $39.0 \pm 3.6$ & $36.4 \pm 2.9$ & $\cdots$ \\
\hline & $\mathbf{F}$ & $\ldots$ & $\cdots$ & $\cdots$ & $25.6 \pm 1.8$ \\
\hline \multirow[t]{2}{*}{2} & $\mathbf{N}$ & $6.9 \pm 1.5$ & $3.1 \pm 1.2$ & $3.5 \pm 1.2$ & $\ldots$ \\
\hline & $\begin{array}{l}\mathrm{D} \\
\mathbf{F}\end{array}$ & $\begin{array}{c}11.4 \pm 1.1 \\
\ldots\end{array}$ & $\begin{array}{c}9.6 \pm 1.8 \\
\ldots\end{array}$ & $\begin{array}{c}10.2 \pm 1.4 \\
\ldots\end{array}$ & $\begin{array}{c}\cdots \\
10.4 \pm 1.1\end{array}$ \\
\hline \multirow{3}{*}{$3 \ddagger$} & $\mathbf{N}$ & $3.1 \pm 1.0$ & $2.0 \pm 1.0$ & $3.0 \pm 1.1$ & $\ldots$ \\
\hline & $\mathrm{D}$ & $2.7 \pm 0.6$ & $2.0 \pm 0.8$ & $3.9 \pm 1.0$ & $\ldots$ \\
\hline & $\mathbf{F}$ & $3.0 \pm 0.5$ & $1.7 \pm 0.4$ & $3.1 \pm 0.6$ & $2.5 \pm 0.6$ \\
\hline \multirow[t]{3}{*}{$4 \ddagger$} & $\mathbf{N}$ & $3.1 \pm 1.0$ & $1.0 \pm 0.7$ & $1.3 \pm 0.7$ & $\cdots$ \\
\hline & D & $2.7 \pm 0.6$ & $3.3 \pm 1.0$ & $1.2 \pm 0.5$ & $\cdots$ \\
\hline & $\mathbf{F}$ & $2.7 \pm 0.5$ & $2.7 \pm 0.6$ & $1.4 \pm 0.4$ & $0.36 \pm 0.21$ \\
\hline \multirow[t]{3}{*}{$\mathrm{P} \S$} & $\mathbf{N}$ & $9.6 \pm 1.8$ & $9.2 \pm 2.2$ & $11.3 \pm 2.2$ & $\cdots$ \\
\hline & D & $9.8 \pm 1.1$ & $10.6 \pm 1.9$ & $9.2 \pm 1.5$ & $\cdots$ \\
\hline & $\mathbf{F}$ & $9.5 \pm 0.8$ & $9.3 \pm 1.1$ & $9.8 \pm 1.1$ & $\cdots$ \\
\hline
\end{tabular}

* N : night interval (after 1710 local time in C1, before 0420 in C2, and before 0540 in C4). D: day interval (before 1500 local time in C1, after 0720 in C2, and after 0820 in C4). F: entire float period, including night, day, and intermediate intervals.

$\dagger$ Type 0 events satisfy all criteria for type 1, except the lack of a Cerenkov signal. They correspond to electrons of approximately 4-12 Mev admixed with an unknown $\gamma$-ray background, which should be constant in time.

$\ddagger$ In flight C4 (modified configuration) the calibration for events of type 3 and 4 is different from corresponding calibrations for other flights. (See paper 1.)

$\$$ Events corresponding to protons above $16 \mathrm{Gev}$, with no expected temporal variation.

transition because the primary cosmic rays responsible for the albedo electrons have rigidity above $0.8 \mathrm{Gv}$, substantially higher than the internal field geomagnetic cutoff along our flight trajectories.

Table 1 summarizes the observed rates for each type of event. (Events of types 2, 3, and 4 correspond to electrons of energies approximately 50 to 100,100 to 300 , and 300 tó 1000 Mev, respectively.) The 'day' and 'night' data were selected to include only time intervals for which both type 1 and type 2 event rates were within one standard deviation of their mean night or day values. The rates in Table 1 show that events of types 0,1 , and 2 have a clear diurnal variation, whereas events of types 3,4 , and $P$ have no statistically significant variation.

\section{Discussion}

Our results clearly show the presence of the diurnal variation of low-energy electron fluxes near Fort Churchill in 1967, which was seen by Jokipii et al. [1967] in 1965 and 1966 and by Webber [1968] in 1966. Our data from flight C1 (see Figure 2) also represent the first observation of the morning and evening transition on the same flight, lending support to the model of Jokipii et al. [1967] and Webber [1968] that their observations of individual morning or evening transitions fit into the pattern of a diurnal variation; i.e., the higher fluxes exist during the whole daytime interval and the lower fluxes exist during the whole nighttime interval and are not restricted to a few hours. As proposed by Jokipii et al. [1967], the diurnal variation of low-energy electron fluxes can be related to a diurnal change in the geomagnetic cutoff, with lower cutoffs prevailing during the nighttime interval. If the nighttime cutoff value lies below the detector threshold, the observed nighttime electron flux at the top of the atmosphere consists of primary particles. During 
the day interval, when a higher cutoff prevails, primaries below cutoff are excluded and return albedo particles with rigidities below cutoff are observed.

Experimental evidence for the diurnal variation of geomagnetic cutoffs at rigidities $\geq 50 \mathrm{Mv}$ has also been derived from satellite observations of low-energy protons [Stone, 1964; Paulikas et al., 1968]. The electron measurements allow us to extend these studies to lower rigidities, below $20 \mathrm{Mv}$. Indirect evidence for a diurnal cutoff variation also comes from observations of asymmetry in the high-latitude boundary of the geomagnetically trapped radiation [Burrows and McDiarmid, 1968].

Our analysis benefits from the important fact that we made measurements both of the diurnally varying downward-moving flux and of the splash albedo. These data were gathered with the same detector, near the same location, within one month, on magnetically quiet days, and so permit a direct comparison between the diurnally varying flux of downward-moving. electrons and the splash albedo electrons near the same location. The mean daytime type 1 rate in flights $\mathrm{C} 1, \mathrm{C} 2$, and $\mathrm{C} 4$ is $35 \pm 1.5$ events/hr. Subtracting our best estimate of the atmospheric secondary contribution (see paper 1) leaves $26.8 \pm 1.5$ events/hr as the rate due to electrons incident at the top of the atmosphere. This value is in good agreement with the corresponding splash albedo rate of $25.6 \pm$ 1.8 events $/ \mathrm{hr}$. The agreement supports the model in which the high daytime flux of electrons is return albedo.

Further support for the model comes from the observation that the nighttime flux is significantly lower than the splash albedo. As shown in paper 1 , the nighttime type 1 event rate is consistent with the rate expected from atmospheric secondaries alone; an upper limit to the contribution from electrons incident at the top of the atmosphere is 3.9 events/hr. By assuming there are no primary electrons, 3.9events/hr value represents an upper limit to the return albedo. Thus, the return albedo at night is less than $15 \%$ of the splash albedo, and, in fact, it may be zero. This lack of return albedo at night over a wide range of local times provides strong support for the model in which the measured nighttime flux (after subtracting atmospheric secondaries) is the full primary flux. We know of no mechanism by which both return albedo and primaries can be excluded from the observations. The lack of return albedo indicates that the splash albedo escapes from the earth; primary particles must be able to travel similar trajectories in the opposite direction and reach the earth.

We derive an upper limit to the nighttime cutoff rigidity (averaged over the nighttime period of observation) in the following manner: We treat the events of type 1 as being electrons with energy between 12 and $50 \mathrm{Mev}$ at the detector. This interval corresponds to 17 to $57 \mathrm{Mev}$ at the top of the atmosphere. We assume that the daytime count rate, after subtracting atmospheric secondaries, represents the flux of return albedo between 17 and $57 \mathrm{Mev}$ and that the corresponding nighttime count rate represents return albedo between $17 \mathrm{Mev}$ and $E_{n}$, the electron energy corresponding to the nighttime cutoff. Let $j(E)$ be the differential energy spectrum of return albedo; $r_{d}$ and $r_{n}$ are the daytime and nighttime count rates for events of type 1 (after subtracting secondaries). Then

$$
\int_{17}^{E_{n}} j(E) d E / \int_{17}^{57} j(E) d E=r_{n} / r_{d}
$$

We take $j(E)=K E^{-1}$ in accordance with the measured splash albedo spectrum below 100 $\mathrm{Mev}$ (see paper 1). This gives

$$
E_{n}=(17 \mathrm{Mev}) \exp \left[\left(r_{n} / r_{d}\right) \ln (57 / 17)\right]
$$

Taking $r_{d}=26.8$ events $/ \mathrm{hr}$ and $r_{n} \leq 3.9$ events/hr, we derive $E_{n} \leq 20 \mathrm{Mev}$.

For flight C4 we may further lower this estimate for the average nighttime cutoff by considering the type 0 events (see Table 1). The existence of a day-night effect indicates that the nighttime cutoff lies somewhere in or below the interval 4 to $12 \mathrm{Mev}$. Since $12 \mathrm{Mev}$ at the detector corresponds to $17 \mathrm{Mev}$ at the top of the atmosphere, we conclude that the average nighttime cutoff rigidity during flight $\mathrm{C} 4$ is below $17 \mathrm{Mv}$.

To estimate the daytime cutoff energy $E_{d}$, we assume that the daytime energy spectrum has a discontinuity at $E_{d}^{\prime}$, the electron energy at the detector corresponding to $E_{a}$ at the top of the atmosphere. Electrons with energy above $E_{d}^{\prime}$ are above the cutoff at all times, day and night, so that we take the total daytime spec- 




Fig. 3. Geomagnetic cutoff rigidity versus geomagnetic dipole latitude. The curves represent calculated vertical cutoff rigidities $\left(R_{0}\right)$. Curve 1 represents internal field, $R_{c}=14.9 \mathrm{Gv} \cos ^{4} \lambda$; curve 2, noon cutoff [Gall et al., 1968] ; curve 3, midnight cutoff [Gall et al., 1968]; curve 4, midnight cutoff [Reid and Sauer, 1967]. The crosses indicate the noon and midnight cutoffs calculated for particles incident parallel to the local field lines [Taylor, 1967]. Experimental results (open symbols representing daytime cutoffs; solid symbols, nighttime cutoffs) are given by circles, this work; triangles, Stone [1964] (horizontal bar indicating the range of latitudes over which the 'polar foot' was observed during the day); diamonds, Paulikas et al. [1968]; squares, Webber [1968]. The calculations assume that the internal field of the earth is a simple dipole; the latitude shown is the dipole latitude. Our experimental points are plotted at a latitude, $\lambda$, defined by $R_{0}=14.9 \mathrm{Gv} \cos ^{4} \lambda$, where $R_{0}$ is the cutoff calculated on the basis of the real internal field [Shea et al., 1968]. For the latitudes considered in this paper, the latitude so derived agrees within $\pm 0.2^{\circ}$ with invariant latitude.

trum (primaries plus atmospheric secondaries) above $E_{d}^{\prime}$ to be the same as the locally measured nighttime spectrum at the same energies (see paper 1). Below $E_{d}^{\prime}$ the daytime spectrum is the sum of return albedo and atmospheric secondaries. We estimate the return albedo spectrum as equal to our measured splash albedo spectrum (see paper 1) and the daytime atmospheric secondary spectrum as equal to the nighttime one. We then calculate, for various values of $E_{a}$, the ratio of daytime to nighttime count rates for each type of electron event. We find that the observed day/night ratio for type 3 events, $1.05 \pm 0.28$, is inconsistent $(>1.5 \sigma)$ with the calculated ratio if $E_{d}>110 \mathrm{Mev}$. On the other hand, the observed day/night ratio of type 2 events in flights $\mathrm{C} 2$ and $\mathrm{C} 4,3.25 \pm$ 0.95 , is inconsistent with the calculated ratio if $E_{d}<70 \mathrm{Mev}$. We thus estimate that the geomagnetic cutoff rigidity averaged over the daytime interval lies between 70 and $110 \mathrm{Mv}$.

In Figure 3 we compare the cutoff rigidities derived above under the assumptions of a sharp cutoff and the equality of the splash and return albedo spectra with the results from other experiments and calculations. The daytime cutoffs observed in this experiment and by Stone [1964] and Webber [1968] are in qualitative agreement with the calculations of Gall et al. [1968], which show a sharp decrease in the daytime cutoff near $70^{\circ}$ latitude. The experimental data show, however, that this decrease occurs near $68^{\circ}$ rather than $70^{\circ}$ as calculated by Gall et al. [1968]. Our nighttime cutoff upper limit, which is valid at latitudes as low as $67^{\circ}$, is in qualitative agreement with the results of Gall et al. [1968] but extends about $2^{\circ}$ lower in latitude than their calculations predict. Our nighttime result is consistent with those of other experimenters and is also consistent with the calculation of Reid and Sauer [1967] and of Taylor [1967].

Although the observations of Jokipii et al. [1967] and Webber [1968] place the morning and evening transitions of low-energy electron fluxes approximately at 0600 and 1800 LT, respectively, we have investigated the dependence of the transition time on geomagnetic latitude. In Figure 4 we represent the trajectories of flights $\mathrm{C} 1, \mathrm{C} 2$, and $\mathrm{C} 4$ in a coordinate system

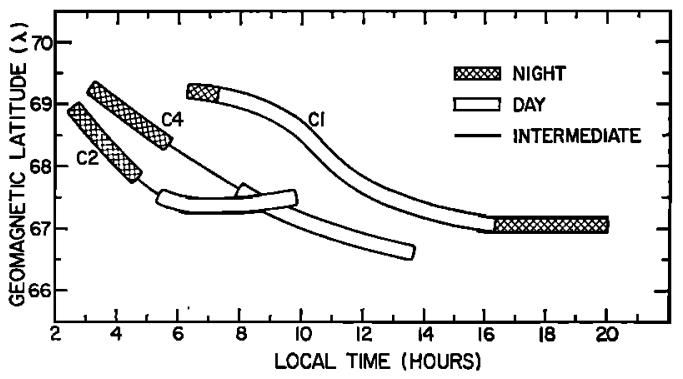

Fig. 4. Latitude and local time of nighttime and daytime count rates for events of type 1 . Latitude is defined by $R_{0}=14.9 \mathrm{Gv} \cos ^{4} \lambda$, where $R_{\sigma}$ is the vertical cutoff based on the earth's internal field only [Shea et al., -1968]. 
of geomagnetic latitude versus local time. Along each trajectory we indicate when the type 1 event rate was within one standard deviation of its day or night value (from Figure 2). It appears that the geomagnetic cutoff remains below $20 \mathrm{Mv}$ later in the morning at higher latitudes. This observation is qualitatively reasonable because at sufficiently high latitudes ( $>70^{\circ}$ in the model of Gall et al. [1968]) the cutoff would remain below $20 \mathrm{Mv}$ at all times.

Our data also indicate an energy dependence of the time of the day-night transition. In Figure 5 we show the rate of type 2 events (nominal 50-100 Mev) in flights $\mathrm{C} 1, \mathrm{C} 2$, and $\mathrm{C} 4$ in addition to the rate of type 1 events (nominal $12-50 \mathrm{Mev})$.

The rate of type 2 events drops to its nighttime value earlier in the evening and returns to the daytime value later in the morning than does the rate of type 1 events. This feature indicates that the change in cutoff from below 20 to near $90 \mathrm{Mv}$ occurs over a period of from 1 to 3 hours. Gall et al. [1968] have calculated the variation of geomagnetic cutoff with time at $67.8^{\circ}$ latitude. As previously noted, their noon and midnight cutoff rigidities do not agree quantitatively with our observations; however,

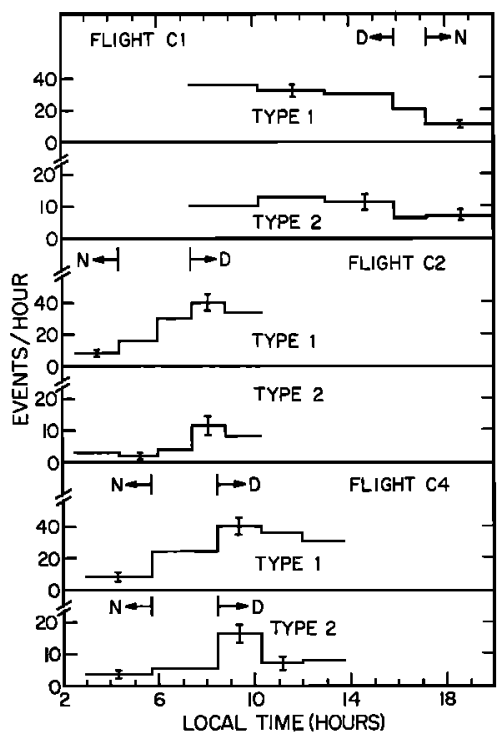

Fig. 5. Type 1 and type 2 event rates versus local time during flights $\mathrm{C1}, \mathrm{C2}$, and C4. Error bars indicate typical statistical errors. $\mathrm{N}$ and $\mathrm{D}$ indicate times during each flight that were included in 'night' and 'day' intervals in Table 1. we again have qualitative agreement, because their calculations show that the change in cutoff rigidity occurs gradually over a period of several hours.

We do not consider the discrepancy between the numerical values of the observed cutoffs and the various calculations to be serious. Each of the calculations uses an idealized model of the magnetosphere, including assumptions for parameters, such as the position and field strength of the neutral sheet, which are not accurately known. Rather, we conclude that the qualitative agreement between the predictions of the theoretical models and the observed characteristics of the low-energy electron flux supports the general features of the models.

Acknowledgments. We profited from stimulating discussions with Professor E. C. Stone.

This work was supported by the National Aeronautics and Space Administration under grant NGL-05-002-007.

\section{ReFerences}

Beedle, R. E., and W. R. Webber, Measurements of cosmic-ray electrons in the energy range 4 Mev to $6 \mathrm{bev}$ at $2 \mathrm{~g} / \mathrm{cm}^{2}$ atmospheric depth at Ft. Churchill, Can. J. Phys., 46, S1014, 1968.

Burrows, J. R., and I. B. MeDiarmid, Local time asymmetries near the high latitude boundary of the outer radiation zone, in Earth's Particles and Fields, p. 45, edited by B. M. McCormac, Reinhold, New York, 1968.

ESSA, Environmental Data Service, Solar and Geophysical Data, IER-FB-282, 158, 1968.

Gall, R., J. Jiménez, and L. Camacho, Arrival of low-energy cosmic rays via the magnetospheric tail, J. Geophys. Res., 79, 1593, 1968.

Israel, M. H., Cosmic-ray electrons between 12 $\mathrm{Mev}$ and $1 \mathrm{Gev}$ in 1967, J. Geophys. Res., 74, this issue, 1969.

Israel, M. H., and R. E. Vogt, Flux of cosmic-ray electrons between 17 and $63 \mathrm{Mev}$, Phys. Rev. Letters, $20,1053,1968$.

Jokipii, J. R., J. L'Heureux, and P. Meyer, Diurnal intensity variation of low-energy electrons observed near the polar cap., J. Geophys. Res., 7Q, $4375,1967$.

L'Heureux, J., The primary cosmic-ray electron spectrum near solar minimum, Astrophys. J., $148,399,1967$.

Lincoln, J. V., Geomagnetic and solar data, $J$. Geophys. Res., 79, 3094, 1968.

Meyer, $P$., and R. Vogt, Electrons in the primary cosmic radiation, Phys. Rev. Letters, 6, 193, 1961.

Paulikas, G. A., J. B. Blake, and S. C. Freden, Low-energy solar-cosmic-ray cutoffs: Diurnal variations and pitch-angle distributions, J. Geophys. Res., 79, 87, 1968. 
Reid, G. C., and H. H. Sauer, The influence of the geomagnetic tail on low-energy cosmic-ray cutoffs, J. Geophys. Res., 7\%, 197, 1967.

Shea, M. A., D. F. Smart, and J. R. McCall, A five degree by fifteen degree world grid of trajectory-determined vertical cutoff rigidities, $C a n$. J. Phys., 46, S1098, 1968.

Stone, E. C., Local time dependence of nonStörmer cutoff for $1.5-\mathrm{Mev}$ protons in quiet geomagnetic field, J. Geophys. Res., 69, 3577, 1964.
Taylor, H. E., Latitude local-time dependence of low-energy cosmic-ray cutoffs in a realistic geomagnetic field, J. Geophys. Res., $7 \mathscr{2}, 4467,1967$.

Webber, W. R., Diurnal variation of the intensity and energy spectrum of low-energy electrons incident at Fort Churchill, Canada, J. Geophys. Res., 73, 4905, 1968.

(Received December 18, 1968; revised June 5, 1969.) 